\title{
Concentrated ERP Delivered in a Group Setting: A Replication Study
}

\author{
Audun Havnen and Bjarne Hansen \\ The OCD-team, Haukeland University Hospital, Bergen, Norway, and Department of Clinical \\ Psychology, University of Bergen, Bergen, Norway
}

\begin{abstract}
Lars-Göran Öst
The OCD-team, Haukeland University Hospital, Bergen, Norway; Department of Psychology, Stockholm University, Stockholm, Sweden, and Department of Clinical Neuroscience, The Karolinska Institute, Stockholm, Sweden
\end{abstract}

\section{Gerd Kvale}

The OCD-team, Haukeland University Hospital, Bergen, Norway, and Department of Clinical Psychology, University of Bergen, Bergen, Norway

\begin{abstract}
Background: In a previous effectiveness study (Havnen et al., 2014), 35 obsessive compulsive disorder (OCD) patients underwent Concentrated Exposure Treatment (cET), which is a newly developed group treatment format delivered over four consecutive days. Aims: The primary aims of the present study were to evaluate the treatment results for a new sample of OCD patients receiving the cET treatment approach and to replicate the effectiveness study described in Havnen et al. (2014). Method: Forty-two OCD patients underwent cET treatment. Treatment was delivered by different therapists than in Havnen et al. (2014), except for two groups led by the developers of the treatment. Assessments of OCD symptom severity, treatment satisfaction, and occupational impairment were included. Results: The results showed a significant reduction in Yale-Brown Obsessive Compulsive Scale scores from pretreatment to post-treatment, which was maintained at 6-month follow-up. At post-treatment, $74 \%$ of the sample was remitted; at 6-month follow-up, $60 \%$ were recovered. The sample showed a very high degree of overall treatment satisfaction. The results from the present study were statistically compared with those obtained in the previous study. The analyses showed that the study samples had comparable demographic data and equal application of treatment. The outcome of the present and original study did not differ significantly on primary and secondary outcome measures. Conclusions: This study shows that cET was successfully replicated in a new patient sample treated by different therapists than the original study. The
\end{abstract}

Correspondence to Audun Havnen, The OCD-team, Haukeland University Hospital, Bergen and Department of Clinical Psychology, University of Bergen, Norway. E-mail: audunhavnen@gmail.com 
results indicate that cET is well accepted by the patients, and the potential for dissemination is discussed.

Keywords: OCD, ERP, group therapy, replication study

\section{Introduction}

Exposure and response prevention (ERP) and cognitive behavioural therapy (CBT) are evidence-based treatments for obsessive compulsive disorder (OCD). In a recently published effectiveness study (Havnen et al., 2014), we demonstrated that a novel format of ERP yields highly encouraging results. The individually tailored and therapist-assisted treatment is delivered in a group setting over four consecutive days, where the ratio between therapists and patients is $1: 1$. The concentrated exposure treatment (cET) is highly accepted by patients and there are few drop-outs. In Havnen et al. (2014), 11\% of patients were improved and 77\% recovered, applying the Jacobson and Truax (1991) criteria, and the comparable figures at 6month follow-up were 14 and $74 \%$, respectively. Furthermore, the cET yielded significant improvement in depressive symptoms and positive changes in occupational interference, which were maintained at follow-up.

The treatment in Havnen et al. (2014) was delivered at an out-patient clinic in the general national health service, has high ecological validity, and has potential for dissemination. The cET also contains several variables that previous research has shown to be of importance for the treatment outcome.

Replication is necessary to ascertain the validity of the previous study on cET. Interestingly, replications are rarely seen in psychological research. The primary aims of the present study were to evaluate the treatment results for a sample of patients receiving the cET treatment approach for OCD, and to replicate the effectiveness study described in Havnen et al. (2014). It will be investigated if treatment results are comparable for a new sample of consecutive patients from the same clinic.

\section{Method}

\section{Participants}

The participants were 42 adults diagnosed with OCD (67\% female, mean age 32.6 years; SD 10.8).

\section{Measures}

Severity of obsessions and compulsions was assessed with the clinician-administered YaleBrown Obsessive Compulsive Scale (Y-BOCS; Goodman et al., 1989). An independent psychologist conducted interviews at post-treatment and follow-up. The Y-BOCS has 10 items and a scoring range of 0 to 40. Treatment satisfaction was assessed with the Client Satisfaction Questionnaire 8 (CSQ-8; Larsen et al., 1979). The CSQ-8 has eight items which are rated on a 1 to 4 scale; total score is 8-32. The Work and Social Adjustment Scale (WSAS; Mundt et al., 2002) was applied to assess work and social impairment. The five items are rated on a scale of 0 to 8 . 


\section{Therapists}

Six clinical psychologists were the therapists. Two of the ten groups were run by the developers of the cET (B.H. and G.K.). A comparison of the therapists in the present study and those in Havnen et al. (2014) showed an overlap of 4.8\%, which was accounted for by the two groups led by the developers. The treatment protocol was identical in the two studies.

\section{Treatment}

The patients received the concentrated exposure treatment (cET; Havnen et al., 2014), conducted over four consecutive days. Day 1 is a 3-hour group session covering psychoeducation and planning of the exposures; days 2 and 3 are full days of therapistassisted exposure work lasting 8-10 h. Exposures are conducted in all settings relevant for each individual, and patients continue exposure on their own after the end of treatment days 2 and 3. On day 4, a 3-4 h session covers core features of the treatment and strategies to prevent or handle setbacks are discussed. The patients make plans for exposure tasks for 3 weeks following treatment. Three months after treatment, patients are invited for a booster session. Treatment is delivered as part of the ordinary psychiatric treatment and patients may request additional sessions if needed.

\section{Comparison between the current and the previous study}

The referral process was identical for the current sample and the sample in Havnen et al. (2014). Comparison of pre-treatment characteristics showed more patients with serotonin reuptake inhibitors (SSRI) in Havnen et al. (2014), whereas remaining variables yielded no significant differences.

Prior to treatment initiation, patients in both samples were instructed to discontinue any use of sleep medication or anxiolytics, and patients using SSRI were instructed to keep medication doses unchanged. All patients in both the current sample and in Havnen et al. (2014) underwent the same treatment procedure.

\section{Statistical analyses}

Statistical analyses on Y-BOCS were analysed with a linear mixed-effects model (LMM). Fixed effects were time (pre- and post-treatment, and 6-month follow-up), co-morbidity and type of previous treatment. Number of additional sessions was included as covariate. Therapist effects were investigated with time, therapist, and time $\mathbf{x}$ therapist interaction as fixed effects. Patients were classified as recovered, improved or unchanged following the Jacobson and Truax (1991) criteria.

\section{Results}

Sixty-two per cent of the sample had co-morbid disorders, with depressive and anxiety disorders being the most prevalent. All patients completed treatment. The primary outcome measure was the Y-BOCS and at post-treatment the sample had a mean Y-BOCS score of 10.8 $(S D=3.9)$ and at 6-month follow-up, mean $12.2(S D=6.4)$. For Y-BOCS the LMM showed 
Table 1. Means and standard deviations on YBOCS

\begin{tabular}{llllllll}
\hline & \multicolumn{3}{c}{ Present sample } & & \multicolumn{3}{c}{ Havnen et al. (2014) } \\
\cline { 2 - 3 } Y-BOCS & Mean & $S D$ & $n$ & & Mean & $S D$ & $n$ \\
\hline Pre-treatment & 25.71 & 4.33 & 42 & & 26.14 & 4.33 & 35 \\
Post-treatment & 10.79 & 3.85 & 42 & & 9.00 & 4.76 & 35 \\
6-month follow-up & 12.21 & 6.42 & 42 & & 10.26 & 5.67 & 35 \\
\hline
\end{tabular}

a significant effect of time; $F(2,126)=120.1, p<.001$, with significant decrease in Y-BOCS score from pre- to post-treatment and pre- to 6-month follow-up. The effects of co-morbidity, previous treatment, number of additional sessions and therapists were not significant. For patients with or without SSRI, a one-way ANOVA showed no significant difference on Y-BOCS.

At post-treatment, $73.8 \%$ of the sample was categorized as recovered, $9.5 \%$ as improved and $16.7 \%$ as unchanged. At 6-month follow-up, the rates were $59.5 \%$ recovered, $16.7 \%$ improved and $23.8 \%$ unchanged.

\section{Comparison between the current and the previous study}

The statistical analyses showed no significant difference in Y-BOCS scores in the present sample and Havnen et al. (2014) at any time point (Table 1). There were no statistical differences in proportion of recovered, improved and unchanged patients in the samples. There was no significant difference between Havnen et al. (2014) and the present study in proportions of patients experiencing a positive change (73\%). In Havnen et al. (2014), 23 of the 27 patients $(85 \%)$ who were recovered at post-treatment, were still classified as recovered at 6-month follow-up. In the present study, 22 of the 31 patients $(71 \%)$ who were recovered at post-treatment, were still recovered at 6-month follow-up. A chi-square test showed no statistical difference in proportion of patients who remained recovered $\left[85 \%\right.$ vs $71 \% ; \chi^{2}$ (1) $=0.96, p=.33]$.

\section{Discussion}

The aims of the present study were to evaluate the treatment results of patients undergoing the cET approach for OCD, and to investigate if the results from a previous effectiveness study (Havnen et al., 2014) from the same clinic could be replicated. Outcomes on Y-BOCS scores were encouraging, with significant reductions in obsessive compulsive symptoms from pre- to post-treatment, and these results were maintained at follow-up assessment. The recovery rate following treatment was high, with $74 \%$ recovered at post-treatment and $60 \%$ recovered at $6-$ month follow-up. There were no significant differences in Y-BOCS scores at any assessment point between the present study and the Havnen et al. (2014) study. Furthermore, the recovery rates in the studies did not differ.

We also tested if there was a difference in treatment outcome in the groups led by the developers of the cET and the groups run by other therapists. The analyses showed that the treatment results were not different between the subgroups at any time point. This finding 
points to the generalizability of the treatment approach, at least across therapists at the same clinic. The next step in the replication process is studies at a different site and this work is currently underway in Norway.

OCD is known to cause functional impairment, thus an important result is the improved employment situation reported by the patients. More than $73 \%$ of the patients described a positive or highly positive development with regard to work or studies, and only one patient described deterioration in the employment situation.

There are several theoretical and practical advantages to delivering treatment in a concentrated format. The most obvious is that patients may have faster symptom reduction and return to work sooner, which clearly has social and economic benefits. Also, when several patients undergo treatment together, group cohesion may be of importance in terms of mutual support and empathy, given the demanding nature of exposure therapy. Likewise, therapists may work together and provide mutual support in difficult exposure situations. Another important aspect of the cET is that the patients undergo treatment over four successive days, which makes it unlikely that between-session set-backs occur. This aspect of the treatment format may in part contribute to the favourable treatment results. No patients dropped out of treatment, which is in contrast to a recent meta-analysis (Öst et al., 2015) which showed an attrition rate of $19.1 \%$ in ERP studies. These results, in combination with the positive selfreported treatment satisfaction, indicate a high treatment acceptance of the treatment format.

In addition, there are theory-based arguments for delivering exposure treatment in a concentrated format. In line with research on inhibitory learning (Craske et al., 2014), it has been suggested that it is of importance to conduct exposure to multiple contexts simultaneously, instead of exposing the patient to one fearful stimulus at a time, and the concentrated format may allow for multiple simultaneous fear cues more effectively than spaced sessions. Research implies that therapeutic outcome is not predicted by the degree to which fear decreases during exposure or the fear level at the end of the session, but rather the degree of inhibitory learning, which is argued to be central to fear extinction (Craske et al., 2014). The cET may provide an ideal platform for such learning as the patients are exposed to multiple anxiety arousing cues simultaneously and throughout several hours during the days of exposure treatment. The 4-day cET format also bears resemblance to the progress in the treatment of other anxiety disorders where brief treatments have demonstrated efficacy.

It might be questioned whether this concentrated treatment is practical and can be implemented in all other regions of the world. This is an empirical question that can only be answered through controlled studies in various regions. It should also be pointed out that the cET does not require a group format. Our clinical experience with cET in individual format is that the effects are equally good. Thus the feasibility of this treatment programme needs to be tested on a much larger scale, and a national study in Norway is now underway.

A central aim of this study was to evaluate if the results of the present effectiveness study replicated the results from Havnen et al. (2014). We conclude that the present study provides a replication of Havnen et al. (2014) for the same type of patients, treated at the same clinic with the same treatment method, but delivered by different therapists under supervision by the treatment developers. 


\section{Limitations}

The present study is part of standard care at an out-patient unit, therefore control conditions were not an option. Concentrated treatment formats involve a high degree of therapist assistance and the possibility for patient expectations needs to be addressed. Studies also show that higher symptom severity is related to better treatment outcome, which may imply that patients tend to seek treatment at peaks of severity, given that OCD may fluctuate in severity over time. In order to control for such potentially confounding variables, a randomized controlled study is needed with assessment of long-term efficacy.

\section{Clinical implications}

The major strength of the present study is the replication of a previous effectiveness study on cET, indicating that concentrated treatment for OCD is a feasible format that can be delivered with comparable results in different samples of patients by different therapists.

\section{Acknowledgements}

The authors acknowledge the Division of Psychiatry, Haukeland University Hospital.

Financial support: No external funding was obtained for this study.

Ethical standards: The authors assert that all procedures contributing to this work comply with the ethical standards of the relevant national and institutional committees on human experimentation and with the Helsinki Declaration of 1975, and its most recent revision. The authors assert that all procedures contributing to this work comply with the ethical standards of the relevant national and institutional guides on the care and use of laboratory animals. This paper uses data collected as part of the standard assessment procedure at the out-patient OCD-clinic in Helse Bergen, Norway. The study was approved by the Personvernombudet, August 5, 2012.

Conflicts of interest: There are no conflicts of interest.

\section{Supplementary material}

An extended version is also available online under the Brief Clinical Report Supplementary Materials tab in the table of contents. Please visit https://doi.org/10.1017/ S1352465817000091.

\section{References}

Craske, M., Treanor, M., Conway, C. C., Zbozinek, T. and Vervliet, B. (2014). Maximizing exposure therapy: an inhibitory learning approach. Behaviour Research and Therapy, 58, 10-23. doi: 10.1016/j.brat.2014.04.006

Goodman, W. K., Price, L. H., Rasmussen, S. A., Mazure, C., Fleischmann, R. L., Hill, C. L., Heninger, G. R. and Charney, D. S. (1989). The Yale-Brown Obsessive Compulsive Scale. I. Development, use, and reliability. Archives of General Psychiatry, 46, 1006-1011. 
Havnen, A., Hansen, B., Öst, L.-G. and Kvale, G. (2014). Concentrated ERP delivered in a group setting: an effectiveness study. Journal of Obsessive-Compulsive and Related Disorders, 3, 319-324. doi: https://doi.org/10.1016/j.jocrd.2014.08.002

Jacobson, N. S. and Truax, P. (1991). Clinical significance: a statistical approach to defining meaningful change in psychotherapy research. Journal of Consulting and Clinical Psychology, 59, $12-19$.

Larsen, D. L., Attkisson, C. C., Hargreaves, W. A. and Nguyen, T. D. (1979). Assessment of client/patient satisfaction: development of a general scale. Evaluation and Program Planning, 2, 197-207. doi: https://doi.org/10.1016/0149-7189(79)90094-6

Mundt, J. C., Marks, I. M., Shear, M. K. and Greist, J. M. (2002). The Work and Social Adjustment Scale: a simple measure of impairment in functioning. British Journal of Psychiatry, 180, 461-464. doi: 10.1192/bjp.180.5.461

Öst, L.-G., Havnen, A., Hansen, B. and Kvale, G. (2015). Cognitive behavioral treatments of obsessive-compulsive disorder. A systematic review and meta-analysis of studies published 19932014. Clinical Psychology Review, 40, 156-169. 